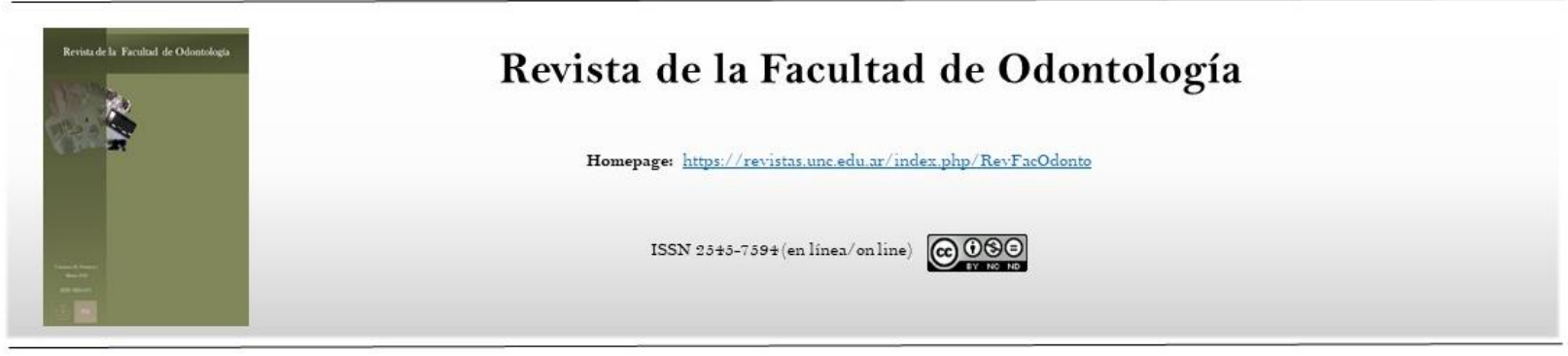

Desafío de la enseñanza clínica de cirugía en modalidad vitual

\title{
Challenge of clinical teaching of surgery in virtual modality
}

Battisti María Eugenia, Ardiles José, Bachur Ricardo

Universidad Nacional de Córdoba. Facultad de Odontología. Departamento de Patología Bucal. Cirugía III

\begin{abstract}
We worked on the design of tools used in a virtual way, adapting the theoretical contents and practical work to the new e-learning modality. In addition, videoconference classes implemented respecting the established schedules and adjusting the times to that context. Through the Moodle platform, and in a virtual classroom of the chair, tutorials, guides and assessment methods were developed, a totally new system for our teaching work
\end{abstract}

KEY WORDS: moodle, virtual teaching, dental surgery

\section{Resumen}

Se trabajó en el diseño de herramientas a utilizar de modo virtual, adaptando los contenidos teóricos y trabajos Prácticos a la nueva modalidad e-learning. Además, se implementaron clases por videoconferencia respetando los horarios establecidos y ajustando los tiempos a ese contexto. A través de la plataforma Moodle, y en un aula virtual de la cátedra, se elaboraron tutoriales, guías y métodos de evaluación, sistema totalmente nuevo para nuestro trabajo docente

PALABRAS CLAVE: Moodle, enseñanza virtual, cirugía dental

Received 7 July 2020; Received in revised form 29 July 2020; Accepted 7 August 2020 
A inicios de este año la cátedra de Cirugía III comenzó, como todos los años, con la planificación y organización de la asignatura y con el dictado de las primeras clases de modo presencial. De modo sorpresivo comenzó a nivel mundial las primeras informaciones sobre una enfermedad denominada COVID-19 que era producida por SARS-COV 2 (del inglés Severe Acute Respiratory Syndrome Coronavirus 2). En marzo la Organización Mundial de la Salud (OMS) declaró la pandemia y el estado argentino declaró cuarentena social, preventiva y obligatoria, medida por la cual se suspendieron todas las actividades presenciales en la UNC ${ }^{1}$.

Esto significó un desconcierto inicial por lo que debíamos afrontar, y de manera acelerada y vertiginosa debimos reorganizar y adecuar la actividad de la Cátedra al nuevo contexto, para que el alumno no se viera perjudicado en su cuatrimestre. Pensar en "virtualizar" la materia, de carácter eminentemente clínico se convirtió en un enorme desafío. El Prof. Dr. Ricardo Bachur comenzó a investigar las plataformas para videoconferencias disponibles, como ZOOM, para el dictado de la materia y a través reuniones virtuales, se empezó a instruir a los docentes en el uso de éstas. Se trabajó en el diseño de herramientas a utilizar de modo virtual, adaptando los contenidos teóricos y trabajos Prácticos a la nueva modalidad e-learning ${ }^{2}$. Además, se implementaron clases por videoconferencia respetando los horarios habituales, y ajustando los tiempos a ese contexto. A través de la plataforma Moodle, y en un aula virtual de la cátedra, se elaboraron tutoriales, guías y métodos de evaluación, sistema totalmente nuevo para nuestro trabajo docente.

Las clases teóricas fueron dictadas por el Prof. Titular y las clases prácticas por los profesores asistentes. Al final de cada clase teórica se respondía las consultas de los alumnos. En las clases teóricas se brindaron los contenidos de la materia. Mientras que, en las clases prácticas, dictadas en comisiones, los docentes pudieron interactuar con los alumnos profundizando el desarrollo de cada unidad temática, e incentivando a los alumnos a la preparación de un trabajo de investigación. Este fue presentado en forma oral a través de ppt a sus compañeros. Para ello se los asesoró y motivó a utilizar los recursos que brindó la Biblioteca de la Facultad. Esta modalidad permitió realizar un acompañamiento de los alumnos que se encontraban desconcertados y ansiosos ante la situación de pandemia, y lograr que las acciones realizadas en los quirófanos, de modo presencial, fueran internalizadas por los alumnos en el modo virtual. El resultado que obtuvimos al finalizar la materia fue óptimo y muy gratificante, destacando el esfuerzo mancomunado de los alumnos y los docentes que pusieron su entusiasmo y responsabilidad en llevar a cabo sus tareas, contando con la asistencia de más del $95 \%$ a todas las actividades programadas por la Cátedra. Fueron opiniones vertidas por los alumnos en una encuesta anónima de evaluación del desempeño docente a través del aula virtual, las que nos hicieron saber que habíamos alcanzado el objetivo planteado.

Los autores declaran que no existen conflictos potenciales de interés con respecto a la autoría y / o publicación de este artículo.

The authors declare no potential conflicts of interest with respect to the authorship and/or publication of this article

\section{Agradecimientos}

Área de Asesoría Docente e Informática de la facultad de Odontología,UNC, Biblioteca de la Facultad de Odontología UNC y docentes de la cátedra de Cirugía III: N. Bachur, C. Bornancini, J. Cima, O. Corominas, M. Giraudo, G. Mordkovich, L. Menutti, L. Bongianino, M. Zalazar, W. Osterode, A. Díaz, M. Pericas, F.Borrego.

\section{Referencias}

1. Rodríguez-Morales AJ, Cardona-Ospina JA, GutiérrezOcampo E, et al. Clinical, laboratory and imaging features of COVID-19: A systematic review and metaanalysis Travel Med Infect Dis. 2020;101623. doi: 10.1016/j.tmaid.2020.101623.

2. Machado RA, Bonan PRF, Perez DEDC, Martelli JH. COVID-19 pandemic and the impact on dental education: discussing current and future perspectives. Braz Oral Res. 2020;34: e083. doi:10.1590/1807-3107bor-2020.vol34.0083

Corresponding to /correspondencia a: Dra. María Eugenia Battisti Cátedra Cirugía III. Departamento de Patología Bucal, Facultad de Odontología, Universidad Nacional de Córdoba. Haya de la Torre s/ n. Pabellón Argentina, Ciudad Universitaria. Córdoba. Argentina. E-mail/ Correo electrónico: eugenia.battisti@unc.edu.ar 